\title{
Improved adsorption energetics within density-functional theory using revised
} Perdew-Burke-Ernzerhof functionals

\author{
Hammer, Bjørk; Hansen, Lars Bruno; Nørskov, Jens Kehlet
}

Published in:

Physical Review B

Link to article, DOI:

10.1103/PhysRevB.59.7413

Publication date:

1999

Document Version

Publisher's PDF, also known as Version of record

Link back to DTU Orbit

Citation (APA):

Hammer, B., Hansen, L. B., \& Nørskov, J. K. (1999). Improved adsorption energetics within density-functional theory using revised Perdew-Burke-Ernzerhof functionals. Physical Review B, 59(11), 7413-7421.

https://doi.org/10.1103/PhysRevB.59.7413

\section{General rights}

Copyright and moral rights for the publications made accessible in the public portal are retained by the authors and/or other copyright owners and it is a condition of accessing publications that users recognise and abide by the legal requirements associated with these rights.

- Users may download and print one copy of any publication from the public portal for the purpose of private study or research.

- You may not further distribute the material or use it for any profit-making activity or commercial gain

- You may freely distribute the URL identifying the publication in the public portal

If you believe that this document breaches copyright please contact us providing details, and we will remove access to the work immediately and investigate your claim 


\title{
Improved adsorption energetics within density-functional theory using revised Perdew-Burke-Ernzerhof functionals
}

\author{
B. Hammer \\ Institute of Physics, Aalborg University, Pontoppidanstrcede 103, DK-9220 Aalborg Dst, Denmark \\ L. B. Hansen and J. K. Nørskov \\ Center for Atomic-scale Materials Physics, Department of Physics, Technical University of Denmark, DK-2800 Lyngby, Denmark
}

(Received 23 September 1998)

\begin{abstract}
A simple formulation of a generalized gradient approximation for the exchange and correlation energy of electrons has been proposed by Perdew, Burke, and Ernzerhof (PBE) [Phys. Rev. Lett. 77, 3865 (1996)]. Subsequently Zhang and Yang [Phys. Rev. Lett. 80, 890 (1998)] have shown that a slight revision of the PBE functional systematically improves the atomization energies for a large database of small molecules. In the present work, we show that the Zhang and Yang functional (revPBE) also improves the chemisorption energetics of atoms and molecules on transition-metal surfaces. Our test systems comprise atomic and molecular adsorption of oxygen, $\mathrm{CO}$, and $\mathrm{NO}$ on $\mathrm{Ni}(100), \mathrm{Ni}(111), \mathrm{Rh}(100), \mathrm{Pd}(100)$, and $\mathrm{Pd}(111)$ surfaces. As the revPBE functional may locally violate the Lieb-Oxford criterion, we further develop an alternative revision of the PBE functional, RPBE, which gives the same improvement of the chemisorption energies as the revPBE functional at the same time as it fulfills the Lieb-Oxford criterion locally. [S0163-1829(99)02711-3]
\end{abstract}

\section{INTRODUCTION}

Density-functional theory is widely accepted as a framework for the study of the electronic ground-state properties of molecules and solids. It has long been realized that the molecular bond energies and the cohesive energies of the solids are overestimated when the electronic exchange and correlation effects are described in the local-density approximation (LDA). ${ }^{1}$ However, the development of nonlocal exchange and correlation functionals has demonstrated that the bond energies of molecules, the cohesive energies of solids, and the energy barriers for molecular reactions can be greatly improved within density-functional theory. ${ }^{2-12}$

In the present paper we investigate the behavior of the chemisorption energy of different atomic and molecular adsorbates on some late transition-metal surfaces using different functionals for the exchange and correlation energy. We limit ourselves to the study of functionals derived in the generalized gradient approximation (GGA). The functionals we use include the Perdew-Wang-91 functional ${ }^{2}$ (PW91), the Perdew-Burke-Ernzerhof (PBE) functional, ${ }^{13}$ and the revised PBE functional with one parameter, $\kappa$, changed from 0.804 to 1.245 (revPBE) as proposed by Zhang and Yang. ${ }^{14}$ These three functionals have been reported to give similar values for the molecular bond energies of about 20 small molecules, with some evidence that the revPBE functional is the most accurate. Our calculations show, however, that the functionals give rather different chemisorption energies. Judging from a comparison to experimental chemisorption energies, the revPBE is found to be superior in the description of the energetics of atomic and molecular bonding to surfaces. The construction of the revPBE functional involves a softening of one of the criteria used in the construction of the PBE functional. Specifically, with the PBE functional, the LiebOxford criterion ${ }^{15,16}$ is obeyed locally and hence also globally by construction, ${ }^{17}$ while with the revPBE functional it is only found empirically to be obeyed globally. ${ }^{14}$ To avoid the uncertainty with respect to the fulfilment of the LiebOxford criterion associated with the use of the revPBE, we develop an alternative functional (RPBE), which gives practically identical chemisorption energies. It does not involve fitting of parameters and it fulfills the Lieb-Oxford criterion by construction.

The paper is organized as follows: First chemisorption energies calculated with the PW91, PBE, and revPBE functionals are presented. Next an analysis of the spatial and gradient-resolved exchange-correlation contributions to the chemisorption energies for the three different functionals is given. This leads to the suggestion of the RPBE functional, which is finally tested.

\section{CALCUlational DETAILS}

The density-functional theory calculations are done for adsorbates in $p(2 \times 2)$ structures on the fcc $(100)$ and fcc(111) faces of $\mathrm{Ni}, \mathrm{Rh}$, and Pd surfaces. The surfaces are modeled by slabs of four and three layers thickness for the two facets (100) and (111), respectively [see the Appendix, issue (iii)]. The slabs are repeated periodically in three dimensions, leaving at least $10 \AA$ of vacuum between the slabs. ${ }^{18}$ The ionic cores are described by ultrasoft pseudopotentials $^{19}$ developed within the PW91 approximation for the exchange and correlation [see the Appendix, issue (ii)]. The nonlinear core correction ${ }^{20}$ is employed, using the core density beyond a cutoff, $r_{c}\left(r_{c}^{C}=0.6, r_{c}^{N}=0.6, r_{c}^{O}\right.$ $\left.=0.7, r_{c}^{N i}=1.2, r_{c}^{R h}=1.0, r_{c}^{P d}=1.1 \mathrm{bohr}\right)$ and a second-order polynomial continuation for smaller $r$ (for the description of oxygen we make one improvement over the above-see issue (i) of the Appendix]. Within the slabs, the PW91 lattice parameters of $3.52,3.83$, and $3.99 \AA$ for $\mathrm{Ni}, \mathrm{Rh}$, and Pd are used throughout the work except when stated otherwise. For $\mathrm{Ni}$, the calculations are done spin polarized. For each slab 
system, the adsorption of atoms and molecules is done on only one of the two slab surfaces exposed and the electrostatic potential is adjusted accordingly. ${ }^{21}$

The Kohn-Sham one-electron valence states are expanded in a basis of plane waves with kinetic energies below $25 \mathrm{Ry}$ at $\mathbf{k}$-point sampling meshes of 64 and $54 \mathbf{k}$ points within the first Brillouin zone for the fcc(100) and fcc(111), respectively. The self-consistent densities are determined by subsequent iterative diagonalization of the Kohn-Sham Hamiltonian, Fermi-population of the Kohn-Sham states $\left(k_{B} T\right.$ $=0.1 \mathrm{eV}$ ), and Pulay mixing of the resulting electronic density. ${ }^{22}$ All total energies have been extrapolated to $k_{B} T$ $=0 \mathrm{eV}$.

The chemisorbed atoms and molecules have been placed in atop, bridge, and hollow [for fcc(111): fcc-hollow] sites and have been relaxed to find the optimum chemisorption energy, while the surface ions have been kept fixed at the truncated bulk positions [see the Appendix, issue (iv)]. For each adsorption system, only results for the most stable adsorption site are reported. The chemisorption energy is calculated as the energy difference:

$$
\begin{gathered}
E_{\text {chem }}=E_{A M}-E_{A}-E_{M}=\sum_{i=A M, A, M} p_{i} E_{i}, \\
p_{A M}=1, \quad p_{A}=p_{M}=-1,
\end{gathered}
$$

where $E_{A M}$ is the total energy of the system of adsorbate $A$ on metal surface $M$, and $E_{A}$ and $E_{M}$ are the total energies of the isolated adsorbate and metal surface, respectively. In the case of dissociative adsorption of a molecule, $A B$, the chemisorption energy is calculated according to

$$
E_{\text {chem }}=E_{A M}+E_{B M}-E_{A B}-2 E_{M} .
$$

The isolated atoms and molecules are treated in very large supercells of dimensions $10.00 \times 10.25 \times 10.50 \AA^{3}$ with $\Gamma$-point sampling of the Brillouin zone. The odd shape of the supercells guarantees that nonspherical densities result where required. ${ }^{23}$

For self-consistently determined chemisorption energies, the exchange-correlation potential entering the Kohn-Sham Hamiltonian is the functional derivative of the exchangecorrelation energy functional evaluated at the ground-state density. The variational principle of density functional theory ${ }^{1,8}$, guarantees, however, that the density and the potential input to the Kohn-Sham Hamiltonian may be varied independently while only giving rise to errors in the total energies that are second order in the variations of the density and potential from their ground-state values. We shall therefore also report on non-self-consistently determined chemisorption energies, where the electron densities $n_{\alpha}$ and ionic coordinates resulting from self-consistent, relaxed calculations with one choice of exchange-correlation potential and energy functional, $\mu_{X C-\alpha}$ and $E_{X C-\alpha}$, are input to different types of exchange-correlation energy functionals, $E_{X C-\beta}$. Such non-self-consistent chemisorption energies are calculated according to

$$
E_{\text {chem }, \beta}=\sum_{i=A M, A, M} p_{i}\left(E_{i}-E_{i, X C-\alpha}\left[n_{\alpha}\right]+E_{i, X C-\beta}\left[n_{\alpha}\right]\right) .
$$

TABLE I. The lattice constant $a$ for bulk Pd and some properties for CO chemisorbed in the fcc-hollow site on $\mathrm{Pd}(111)$ calculated self-consistently with different exchange-correlation functionals. $Z$ is the separation of the $\mathrm{CO}$ center of mass from the outermost $\operatorname{Pd}(111)$ layer, $b$ is the $\mathrm{CO}$ bond length upon adsorption, and $E_{\text {chem }}$ is the chemisorption energy.

\begin{tabular}{lcccccc}
\hline \hline & & LDA & PW91 & PBE & revPBE & RPBE \\
\hline & & & & & & \\
$a$ & $\AA$ & 3.89 & 3.99 & 3.99 & 4.01 & 4.02 \\
$Z$ & $\AA$ & 1.85 & 1.93 & 1.93 & 1.95 & 1.95 \\
$b$ & $\AA$ & 1.19 & 1.19 & 1.19 & 1.19 & 1.19 \\
$E_{\text {chem }}$ & $\mathrm{eV}$ & -2.74 & -2.07 & -1.94 & -1.64 & -1.65 \\
\hline \hline
\end{tabular}

For $\alpha$ and $\beta$ we will use four different functionals from the literature: $\mathrm{LDA},{ }^{25} \mathrm{PW} 91,{ }^{2} \mathrm{PBE},{ }^{13}$ and revPBE, ${ }^{14}$ and the new functional, RPBE, described below. For the four nonlocal GGA functionals, PW91, PBE, revPBE, and RPBE, the exchange-correlation potential is constructed following White and Bird. ${ }^{24}$

The accuracy of the physical and numerical approximations made with the present calculational setup is discussed in the Appendix.

\section{RESULTS AND DISCUSSION}

We start by considering one chemisorption system, $\mathrm{CO}$ adsorption in the fcc hollow on $\operatorname{Pd}(111)$, in some detail. Table I summarizes the structural and energetic results for the system as computed fully self-consistently within the various exchange-correlation approximations considered. The distance $Z$ of the center of mass of the chemisorbed $\mathrm{CO}$ from the outermost surface layer, and the bond length $b$ of the chemisorbed $\mathrm{CO}$, have apparently only little dependence on the choice of nonlocal exchange-correlation functional. $Z$ is, however, somewhat smaller when using local exchangecorrelation (LDA), which is consistent with the LDA also favoring a smaller lattice constant, $a \cdot^{3-7}$ Despite the small change in structural properties, the chemisorption energy $E_{\text {chem }}$ of the CO depends strongly on the choice of exchange-correlation functional. With the various GGA functionals considered, $E_{\text {chem }}$ varies by as much as $0.4 \mathrm{eV}$ (approximately 20\%) and when comparing the result of the LDA calculation with the set of GGA calculations, the difference is of the order of $1 \mathrm{eV}$.

The large differences in chemisorption energies found in the fully self-consistent calculations remain even when the exchange-correlation energies are evaluated only non-selfconsistently, according to Eq. (3). This is clear from the upper half of Table II, which shows such non-self-consistent chemisorption energies $E_{c h e m, \beta}$ as the regular entries and the self-consistent ones as the highlighted entries. Each column of the table gives chemisorption energies with one choice of $\beta$, i.e., one $E_{X C-\beta}$, cf. Eq. (3). Comparing down a column, it is seen that the self-consistently and non-self-consistently determined chemisorption energies differ somewhat less than the $E_{c h e m}$ for different $E_{X C-\beta}$ functionals. This is a consequence of the variational principle as discussed above. The only noteworthy errors in the non-self-consistent $E_{\text {chem }}$ arise when an LDA density is input to a GGA $E_{X C}$ functional or 
TABLE II. Non-self-consistent chemisorption energies, Eq. (3), for $\mathrm{CO} / \mathrm{Pd}(111)$ as a function of the input density $n_{\alpha}$ and the $\mathrm{Pd}$ lattice constant $a$ (see Table I). In the upper half of the table, the lattice constant and hence the volume is chosen to be $a_{\alpha}$, i.e., consistent with the density, while in the lower half of the table, the volume is kept fixed at the PW91 value. The highlighted entries in the upper half are the self-consistent chemisorption energies, while the highlighted entries in the lower half are the approximated values to be used in the remainder of the paper. The two sets of highlighted chemisorption energies are very similar.

\begin{tabular}{lllll}
\hline \hline \multicolumn{4}{c}{$E_{\text {chem }, \beta}$} \\
LDA & PW91 & PBE & revPBE & RPBE \\
\hline
\end{tabular}

\begin{tabular}{lcccccc}
\multicolumn{7}{c}{ Self-consistent volume } \\
$n_{L D A}$ & $a_{L D A}$ & $\mathbf{- 2 . 7 4}$ & -1.80 & -1.69 & -1.30 & -1.28 \\
$n_{P W 91}$ & $a_{P W 91}$ & -2.95 & $\mathbf{- 2 . 0 7}$ & -1.96 & -1.59 & -1.56 \\
$n_{P B E}$ & $a_{P B E}$ & -2.94 & -2.06 & $\mathbf{- 1 . 9 4}$ & -1.57 & -1.55 \\
$n_{\text {revPBE }}$ & $a_{\text {revPBE }}$ & -2.98 & -2.12 & -2.00 & $\mathbf{- 1 . 6 4}$ & -1.62 \\
$n_{R P B E}$ & $a_{R P B E}$ & -3.00 & -2.15 & -2.03 & -1.67 & $\mathbf{- 1 . 6 5}$ \\
& \multicolumn{7}{c}{} \\
& $a_{P W 91}$ & -3.01 & -2.05 & -1.93 & -1.55 & -1.52 \\
$n_{L D A}$ & $a_{P W 91}$ & $\mathbf{- 2 . 9 5}$ & $\mathbf{- 2 . 0 7}$ & $\mathbf{- 1 . 9 6}$ & $\mathbf{- 1 . 5 9}$ & $\mathbf{- 1 . 5 6}$ \\
$n_{P W 91}$ & $a_{P W 91}$ & -2.94 & -2.06 & -1.94 & -1.57 & -1.55 \\
$n_{P B E}$ & $a_{P W 91}$ & -2.92 & -2.06 & -1.94 & -1.58 & -1.55 \\
$n_{r e v P B E}$ & $a_{P W 91}$ & -2.92 & -2.06 & -1.94 & -1.58 & -1.55 \\
$n_{R P B E}$ & &
\end{tabular}

vice versa, i.e., when $E_{\text {chem, } G G A}\left[n_{L D A}\right]$ or $E_{\text {chem, } L D A}\left[n_{G G A}\right]$ are evaluated. At first glance, this suggests that the densities resulting from self-consistent LDA and GGA calculations differ somewhat. That this is probably not the case can be deduced from the lower half of Table II. Here are given the non-self-consistent chemisorption energies that have all been evaluated on the basis of self-consistent calculations done using the PW91 value for the Pd lattice constant $a_{P W 91}$.

The non-self-consistent chemisorption energies at the PW91 volume given in Table II are seen for each $\beta$ to be practically independent of the exchange-correlation functional used in the underlying self-consistent calculations. This is the case even when LDA densities are used as input for a non-self-consistent GGA chemisorption energy, and shows that the densities are indeed very similar in the LDA and the GGA's. It is thus the usage of a volume different from the equilibrium one, which causes most of the error in a non-self-consistent calculation. The volumes resulting from the GGA's presently considered are, however, very similar (cf. Table I) and working with any of the self-consistent GGA volumes will therefore result in small errors in nonself-consistent GGA calculations. In the remainder of the paper, we therefore choose to report only on chemisorption energies for the various exchange-correlation functionals calculated non-self-consistently on the basis of self-consistent PW91 calculations at the PW91 volume. For CO/Pd(111) these values have been highlighted in the lower half of Table II and comparing with the self-consistent values highlighted in the upper half of the table, we judge that the usage of non-self-consistent chemisorption energies evaluated at constant volume can be made without a notable loss of accuracy.

In Table III calculated chemisorption energies are pre-
TABLE III. Calculated chemisorption energies as a function of the exchange-correlation energy functional $\beta$ compared to measured chemisorption energies. All numbers are in $\mathrm{eV}$ per adsorbate. The rms deviations for the calculated chemisorption energies for $\mathrm{O}$, $\mathrm{CO}$, and $\mathrm{NO}, \sigma_{O}, \sigma_{C O}$, and $\sigma_{N O}$, and for all three adsorbates, $\sigma_{\text {tot }}$, have been compiled against the experimental numbers from Refs. 26 and 27 only. The $\sigma$ values in parenthesis arise when the $\mathrm{CO} / \mathrm{Rh}(100)$ data is neglected. The $E_{\text {chem }}^{\exp }$ for $\mathrm{O}$ have been evaluated as $\frac{1}{2}\left\{E_{\text {chem }}^{\text {exp }}\left(\mathrm{O}_{2}\right)-E_{b}^{\text {exp }}\left(\mathrm{O}_{2}\right)\right\}$ with the value $5.11 \mathrm{eV}$ (117.96 $\left.\mathrm{kcal} / \mathrm{mol}^{51}\right)$ for the $\mathrm{O}_{2}$ bond energy $E_{b}^{\exp }\left(\mathrm{O}_{2}\right)$.

\begin{tabular}{cccc}
\multicolumn{2}{c}{$E_{\text {chem, } \beta}$} & $E_{\text {chem }}^{\text {exp }}$ \\
LDA PW91 & PBE revPBE RPBE & \\
\hline
\end{tabular}

\begin{tabular}{lcccccc}
$\mathrm{O}(\mathrm{fcc}) / \mathrm{Ni}(111)$ & -6.68 & -5.38 & -5.27 & -4.83 & -4.77 & $-4.84^{\mathrm{a}}$ \\
$\mathrm{O}(\mathrm{hol}) / \mathrm{Ni}(100)$ & -6.97 & -5.66 & -5.55 & -5.10 & -5.03 & $-5.41^{\mathrm{a}}$ \\
$\mathrm{O}(\mathrm{hol}) / \mathrm{Rh}(100)$ & -6.64 & -5.34 & -5.23 & -4.77 & -4.71 & $-4.56^{\mathrm{a}}$ \\
$\mathrm{O}(\mathrm{fcc}) / \mathrm{Pd}(111)$ & -5.34 & -4.08 & -3.98 & -3.54 & -3.49 & \\
$\mathrm{O}(\mathrm{hol}) / \mathrm{Pd}(100)$ & -5.39 & -4.14 & -4.04 & -3.59 & -3.53 & \\
& & & & & & \\
$\sigma_{O}$ & 1.84 & 0.57 & 0.47 & 0.22 & 0.24 & \\
\hline & & & & & & \\
$\mathrm{CO}(\mathrm{fcc}) / \mathrm{Ni}(111)$ & -2.85 & -1.99 & -1.88 & -1.52 & -1.49 & $-1.35^{\mathrm{a}}$ \\
$\mathrm{CO}(\mathrm{hol}) / \mathrm{Ni}(100)$ & -3.05 & -2.11 & -2.00 & -1.62 & -1.58 & $-1.26^{\mathrm{a}}$ \\
$\mathrm{CO}(\mathrm{brd}) / \mathrm{Rh}(100)$ & -3.02 & -2.28 & -2.16 & -1.84 & -1.81 & $-1.19^{\mathrm{a}}$ \\
$\mathrm{CO}(\mathrm{fcc}) / \mathrm{Pd}(111)$ & -2.95 & -2.07 & -1.96 & -1.59 & -1.56 & $(-1.47)^{\mathrm{b}}$ \\
$\mathrm{CO}(\mathrm{brd}) / \mathrm{Pd}(100)$ & -2.77 & -1.98 & -1.87 & -1.53 & -1.50 & $-1.69^{\mathrm{a}}$ \\
& & & & & & \\
$\sigma_{C O}$ & 1.58 & 0.78 & 0.67 & 0.39 & 0.37 & \\
& $(1.49)$ & $(0.64)$ & $(0.54)$ & $(0.25)$ & $(0.23)$ & \\
\hline
\end{tabular}

\begin{tabular}{lcccccc}
$\mathrm{NO}(\mathrm{hol}) * / \mathrm{Ni}(100)$ & -6.31 & -4.52 & -4.41 & -3.79 & -3.68 & $-3.99^{\mathrm{a}}$ \\
$\mathrm{NO}(\mathrm{brd}) / \mathrm{Rh}(100)$ & -3.73 & -2.76 & -2.67 & -2.31 & -2.28 & \\
$\mathrm{NO}(\mathrm{fcc}) / \mathrm{Pd}(111)$ & -3.27 & -2.20 & -2.12 & -1.72 & -1.67 & $(-1.86)^{\mathrm{c}}$ \\
$\mathrm{NO}(\mathrm{hol}) / \mathrm{Pd}(100)$ & -3.19 & -2.12 & -2.04 & -1.63 & -1.58 & $-1.61^{\mathrm{d}}$ \\
& & & & & & \\
$\sigma_{N O}$ & 1.98 & 0.52 & 0.43 & 0.14 & 0.22 & \\
\hline & & & & & & \\
$\sigma_{\text {tot }}$ & 1.76 & 0.66 & 0.56 & 0.30 & 0.30 & \\
& $(1.76)$ & $(0.58)$ & $(0.48)$ & $(0.21)$ & $(0.23)$ & \\
\hline \hline
\end{tabular}

*Dissociative adsorption.

${ }^{\mathrm{a}} \mathrm{MC}$ experiments reviewed by Brown, Kose, and King, Ref. 26.

${ }^{\mathrm{b}}$ TPD experiment by Conrad et al., Ref. 49.

${ }^{\mathrm{c}}$ TPD experiment by Ramsier et al., Ref. 50

${ }^{\mathrm{d}} \mathrm{MC}$ experiment by Yeo, Vattuone, and King, Ref. 27.

sented for a range of different adsorption systems, using the self-consistent PW91 density and volume. In order to allow for an assessment of the overall quality of the calculated chemisorption energies we include in the table-where available - the experimental initial chemisorption energies from the microcalorimetric (MC) measurements reviewed by Brown, Kose, and King ${ }^{26,27}$ [see the Appendix, issue (v)]. We consider these values to represent a most reliable set of experimental chemisorption energies and we therefore compile the root-mean-square (rms) deviations $\sigma_{O}, \sigma_{C O}$, and $\sigma_{N O}$ for the calculated chemisorption energies with respect to the measured ones for $\mathrm{O}, \mathrm{CO}$, and $\mathrm{NO}$ chemisorption, 
respectively, and $\sigma_{\text {tot }}$ for all adsorption systems considered.

It appears clearly from the table that the LDA functional gives adsorbates overbound by more than $1.5 \mathrm{eV}$. The PW91 and PBE functionals, on the other hand, give more moderate chemisorption energies, which, however, are still too large numerically by about $0.6 \mathrm{eV}$ per adsorbate. Finally, the revPBE functional and the RPBE functional (to be discussed below) prove rather accurate in the description of the chemisorption energies for the present set of adsorbate-metal systems. For these functionals, the typical discrepancy between the theoretical and experimental chemisorption energies is of the order $0.30 \mathrm{eV}$. We note that regardless of which exchange-correlation functional is considered, in particular, the $\mathrm{CO} / \mathrm{Rh}(100)$ system gives rise the the large rms deviations. This raises some question about quality of this one experimental value of $1.19 \mathrm{eV}$ for the initital chemisorption energy. The value further compares poorly with the temperature programmed desorption (TPD) and isothermal desorption experiments in Ref. 28, which are reported to give values for the saturation chemisorption energy around $1.42 \mathrm{eV}$. Values for $\sigma_{C O}$ and $\sigma_{t o t}$ evaluated without the $\mathrm{CO} / \mathrm{Rh}(100)$ system are included in the table in parentheses. It is seen that neglecting the $\mathrm{CO} / \mathrm{Rh}(100)$ system the $\sigma_{\text {tot }}$ would become approximately $0.5 \mathrm{eV}$ for the PW91 and PBE functionals and less than $0.25 \mathrm{eV}$ for the revPBE and RPBE functionals. We note that the uncertainty of the measurements of the chemisorption energies is of the order $0.2 \mathrm{eV}$ and $0.1 \mathrm{eV}$ for atomic and molecular chemisorption, respectively. ${ }^{26}$

The finding of large overbinding of adsorbates to transition-metal surfaces in the LDA and less so in the GGA, e.g., represented by the PW91, was realized early on ${ }^{8-12}$ and many groups are now routinely using the GGA functionals for such surface studies. ${ }^{29-40}$ Besides the evidence for the improvement of the chemisorption energetics with the revPBE presented in this work, there is at present one example where the revPBE has been used for a chemisorption study $-\mathrm{N}_{2}$ adsorption and dissociation on $\mathrm{Fe}(111)$ (Ref. 41) — in which case similar improvements were found. Several suggestions for the reason for the reduction in the overbinding have been put forward, the simplest of which is the following: ${ }^{42}$ (i) GGA functionals favor reduced density gradients, $s \propto|\nabla \rho| / \rho^{4 / 3}$; (ii) the volume of space with large $s$ values scales with the free surface area, where "surface", may represent both a solid surface and the surface of molecules and atoms; (iii) a system of a molecule chemisorbed on a solid surface exposes less surface (and therefore has less volume with large $s$ values) than do the reference systems of a clean solid surface and a gas phase molecule; (iv) consequently, the adsorption system is destabilized over the reference system-i.e., the chemisorption will become less attractive and the chemisorption energy will become less negative-when using a GGA. The loss of surface and hence loss of regions with large reduced density gradients in a chemisorption event is illustrated schematically in Fig. 1.

Some evidence for this simple picture of the effect of the GGA functionals on a chemisorption system can be gained from Fig. 2. In Fig. 2(a) is plotted for $\mathrm{CO} / \mathrm{Pd}(100)$, the gradient-dependent contributions in the GGA to the chemisorption energy:

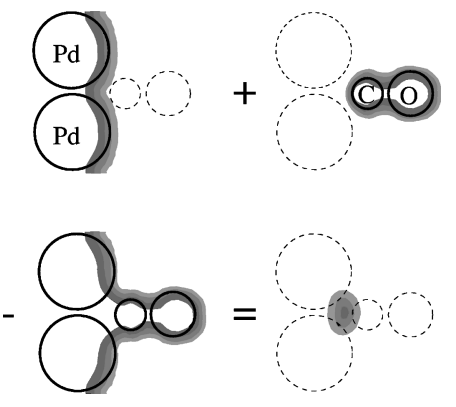

FIG. 1. Outside a solid surface, e.g., Pd, and all round a molecule, e.g. CO, the electron density falls off. These regions of "surface," here shown shaded, have thus large reduced density gradients, $s \propto|\nabla \rho| / \rho^{4 / 3}$. Once a chemisorption bond between the solid surface and the molecule is formed less surface results. The loss of surface appears in the region between the solid surface and the chemisorbed molecule.

$$
\Delta e_{\text {chem }, G G A}(\mathbf{r})=\sum_{i=A M, A, M} p_{i} n_{i}(\mathbf{r})\left[\epsilon_{i, G G A}(\mathbf{r})-\epsilon_{i, L D A}(\mathbf{r})\right],
$$

where $n_{i}(\mathbf{r}) \epsilon_{i, X C}(\mathbf{r})$ is the XC energy density (evaluated locally, $\mathrm{XC}=\mathrm{LDA}$, or nonlocally, $\mathrm{XC}=\mathrm{GGA}$ ) at $\mathbf{r}$ for each of the systems, $i=A M, A, M$. A plane perpendicular to the $\mathrm{Pd}(100)$ surface, through two $\mathrm{Pd}$ atoms in the surface and through a bridge-bonded $\mathrm{CO}$ molecule at these $\mathrm{Pd}$ atoms is chosen. It is apparent that, in this plane, the change in the chemisorption energy comes from the region of contact between the molecule of the surface, i.e., from the region of "loss of surface area"' caused by the adsorption. One should,

a)
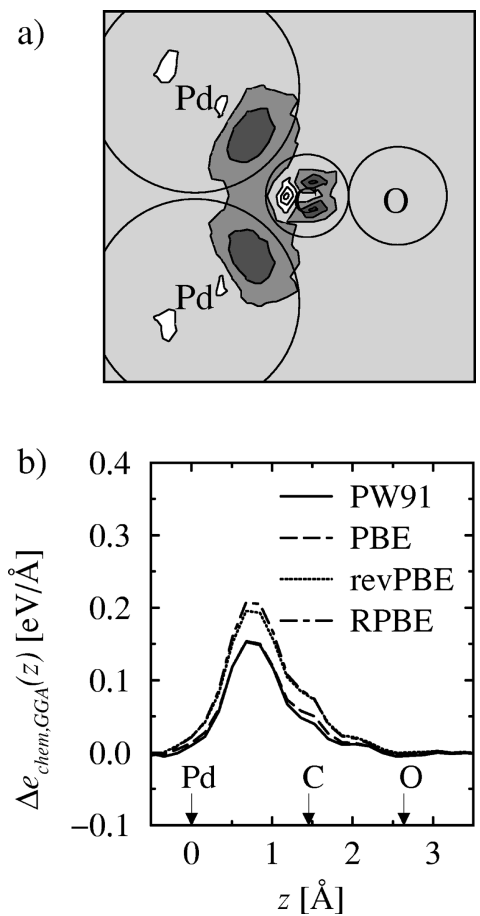

FIG. 2. (a) The spatially resolved contribution to the chemisorption energy from nonlocal exchange-correlation, $\Delta e_{\text {chem, } P W 91}(\mathbf{r})$ for $\mathrm{CO}$ adsorption on $\mathrm{Pd}(100)$. The contours are separated by 0.19 $\mathrm{eV} / \AA^{3}$. (b) The same quantity, but layer integrated, $\Delta e_{\text {chem, } G G A}(z)$ plotted for the different GGA's considered. 
however, note ${ }^{43}$ that the gradient-dependent exchangecorrelation energy density, $\Delta e_{\text {chem, } G G A}(\mathbf{r})$, does not have a physical meaning as such except when integrated over all space:

$$
E_{\text {chem }, G G A}-E_{\text {chem }, L D A}=\int \Delta e_{\text {chem }, G G A}(\mathbf{r}) d \mathbf{r}
$$

where it gives the net effect of the nonlocal exchange and correlation on the chemisorption energy - a term that can also be deduced from Table III. In Fig. 2(b), the nonlocal contribution to the chemisorption energy in each of the four GGA's considered has been integrated over the $p(2 \times 2)$ unit cell area $A$ parallel to the $\operatorname{Pd}(100)$ surface and is plotted as a function of the distance from the outermost layer of $\mathrm{Pd}$ atoms:

$$
\Delta e_{\text {chem }, G G A}(z)=\int_{A} \Delta e_{\text {chem }, G G A}(\mathbf{r}) d x d y .
$$

From this quantity, it is seen that the change in the chemisorption energy is caused by changes in the exchange correlation energy contributions from a region in space highly localized between the solid surface and the molecule. It further appears from the figure that the difference in chemisorption energies determined with the four GGA's considered comes about as merely a scaling of the gradient-dependent exchange correlation energy contributions.

The PW91 and PBE functionals are seen in Fig. 2(b) to have very similar gradient-dependent exchange correlation energy contributions, which causes the very similar chemisorption energies given in Table III. This is to be expected as the PBE functional has been developed to mimic the PW91 functional, but with fewer parameters and a simpler functional form. ${ }^{13}$ The revPBE functional, on the other hand, deliberately deviates from the PBE functional with one parameter $\kappa$ that has been changed to improve the description of atomic total energies and molecular atomization energies. ${ }^{14}$ The parameter $\kappa$ appears in the exchange energy functional:

$$
E_{X}[n]=\int n(\mathbf{r}) \epsilon_{X}(\mathbf{r}) d \mathbf{r}=\int n(\mathbf{r}) \epsilon_{X}^{L D A}(n(\mathbf{r})) F_{X}(s(\mathbf{r})) d \mathbf{r}
$$

where $n(\mathbf{r}) \epsilon_{X}^{L D A}(\mathbf{r})$ is the local exchange energy density from the LDA, $s$ is the reduced density gradient:

$$
s(\mathbf{r})=|\nabla n(\mathbf{r})| /\left[2\left(3 \pi^{2}\right)^{1 / 3} n(\mathbf{r})^{4 / 3}\right],
$$

and $F_{X}$ is a local exchange enhancement factor

$$
F_{X}(s)=1+\kappa-\frac{\kappa}{1+\mu s^{2} / \kappa}
$$

where $\mu$ and $\kappa$ are constants. In the PBE a value $\kappa=0.804$ is used while in the revPBE $\kappa=1.245$ is used. The two enhancement functions $F_{X}(s)$ are plotted in Fig. 3(a). The PBE and revPBE functionals are identical otherwise, i.e., also with respect to the correlation energy terms not given here.

The reasoning of Perdew, Burke, and Ernzerhof, ${ }^{13}$ behind their use of $\kappa=0.804$ in the PBE functional is that $F_{X}(s)$ thereby becomes upper bound by 1.804 and the exchange
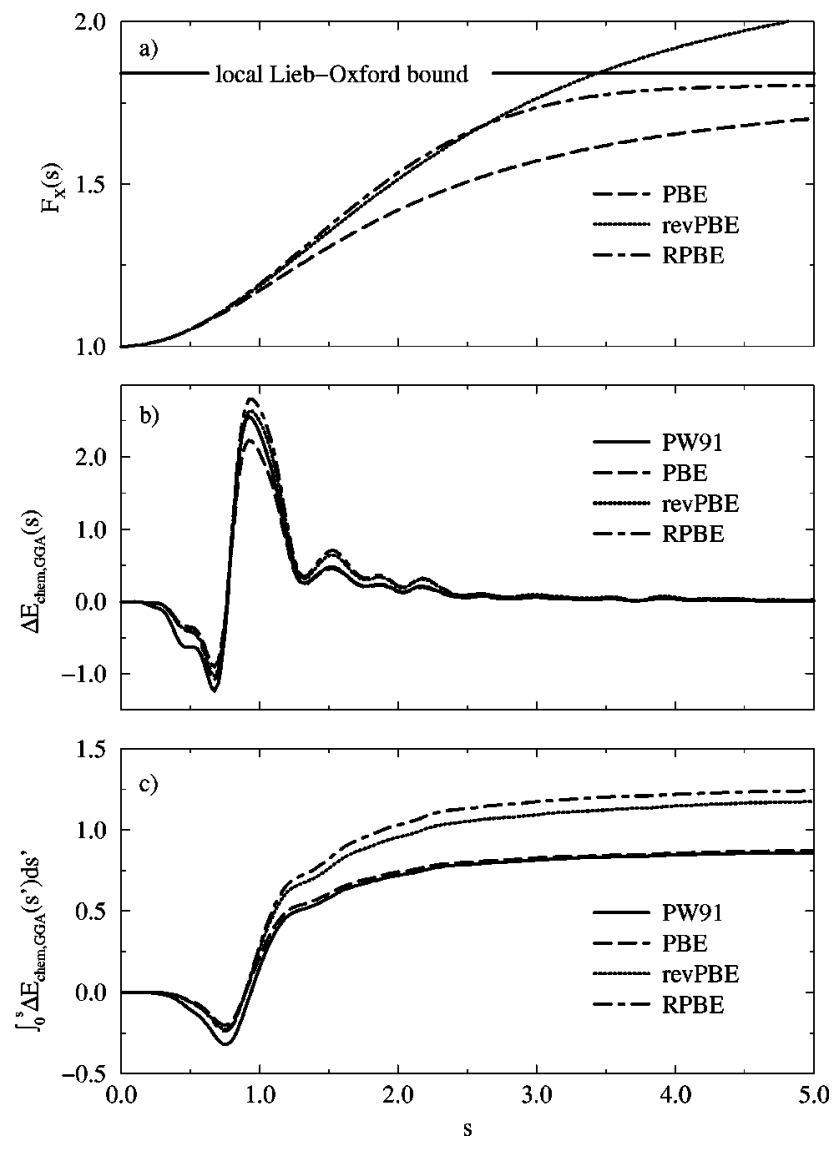

FIG. 3. (a) The exchange enhancement factor for the different GGA functionals as a function of the reduced density gradient, $s$. (b) The distribution of nonlocal exchange-correlation energy contributions, $\Delta E_{\text {chem, } G G A}(s)$, to the chemisorption energy of $\mathrm{CO}$ on $\operatorname{Pd}(100)$. (c) The integrated distribution of nonlocal exchangecorrelation energy contributions to the chemisorption energy of $\mathrm{CO}$ on $\operatorname{Pd}(100)$.

energy is therefore in every point in space subject to the following inequality (using atomic units):

$$
n(\mathbf{r}) \epsilon_{X}(\mathbf{r}) \geqslant-1.679 n(\mathbf{r})^{4 / 3} .
$$

With this criterion on $\epsilon_{X}(\mathbf{r})$, the Lieb-Oxford bound, ${ }^{15,16}$

$$
E_{X}[n] \geqslant E_{X C}[n] \geqslant-1.679 \int n(\mathbf{r})^{4 / 3} d \mathbf{r},
$$

automatically becomes fulfilled. In the construction of the revPBE, Zhang and Yang ${ }^{14}$ point out that for a given electron density a fulfilment of Eq. (10), which may be considered a local Lieb-Oxford bound, is only a sufficient but not a necessary requirement for the fulfilment of the true, integrated Lieb-Oxford bound, Eq. (11). In the revPBE functional $\kappa$ $=1.245$ is therefore chosen and it is reported that the LiebOxford bound is always fulfilled in practice. ${ }^{14}$ However, as stressed by Perdew, Burke, and Ernzerhof ${ }^{17}$ employing the local bound in the GGA construction ensures that the integrated bound will be satisfied for any possible electron density. In the present calculations for atoms and molecules adsorbed on transition-metal surfaces we also find that the Lieb-Oxford bound is fulfilled when using the revPBE functional. 
Considering the great improvement reported in Table II of the chemisorption energies resulting from the slight change in the functional from $\mathrm{PBE}$ to revPBE, it is interesting to trace which values of the reduced gradient $s$ give rise to the improvement. Such an analysis has been done by Zupan et al. for molecular and solid systems. ${ }^{42}$ To do so for a chemisorption system we plot in Figs. 3(b) and 3(c) for $\mathrm{CO} /$ $\operatorname{Pd}(100)$ the $s$-resolved nonlocal exchange correlation contributions to the change in the chemisorption energy (relative to the LDA):

$$
\begin{aligned}
& \Delta E_{\text {chem }, G G A}(s) \\
& =\int \sum_{i=A M, A, M} p_{i} n_{i}(\mathbf{r})\left\{\epsilon_{i, G G A}(\mathbf{r})\right. \\
& \left.\quad-\epsilon_{i, L D A}(\mathbf{r})\right\} \delta\left(s-s_{i}(\mathbf{r})\right) d \mathbf{r},
\end{aligned}
$$

and

$$
\int_{0}^{s} \Delta E_{\text {chem, } G G A}\left(s^{\prime}\right) d s^{\prime} .
$$

When integrated over all $s$, the quantity $\Delta E_{\text {chem, } G G A}(s)$ gives the chemisorption energy change due to the use of a nonlocal exchange-correlation functional [cf. Eq. (5)]:

$$
E_{\text {chem }, G G A}-E_{\text {chem }, L D A}=\int_{0}^{\infty} \Delta E_{\text {chem }, G G A}(s) d s .
$$

From Figs. 3(b) and 3(c) it can be seen that the change in the chemisorption energy from the LDA to a GGA mainly originates from $s$ values between 0.5 and 2.5. Specifically, the non-negligible differences between $\Delta E_{\text {chem,PBE }}(s)$ and $\Delta E_{\text {chem, revPBE }}(s)$ can be seen to lie in this range also. It is interesting to note from Fig. 3(a) that in the $0.5<s<2.5$ range the exchange-enhancement factor, $F_{X}(s)$, still fulfills the local Lieb-Oxford bound, when formulated as $F_{X}(s)$ $\leqslant 1.804$. $^{13}$ This suggests that it should be possible to construct from the PBE a new GGA functional, that changes the chemisorption energies as does the revPBE, but without violating the local Lieb-Oxford bound. A means of doing this is to use the PBE, but choosing for the exchange enhancement factor $F_{X}(s)$ another functional form so that it follows the revPBE exchange enhancement factor only for $s$ values up to 2.5. For larger values of $s$, the function $F_{x}(s)$ should level off, to fulfill $F_{X}(s) \leqslant 1.804$. Choosing

$$
F_{X}(s)=1+\kappa\left(1-e^{-\mu s^{2} / \kappa}\right)
$$

with $\kappa=0.804$ for the exchange enhancement factor one obtains such a functional. The functional is a slight revision of the PBE functional and we denote it the RPBE functional. The FORTRAN computer code required to run with the new functional is available on the internet. ${ }^{44}$ The exchange enhancement factor of the RPBE is plotted in Fig. 3(a). It is constructed to have the same behavior for small $s$ :

$$
F_{x}(s) \rightarrow 1+\mu s^{2}, \quad \text { for } \quad s \rightarrow 0,
$$

as $F_{X}(s)$ from $\mathrm{PBE}$ and revPBE, it grows as rapidly with $s$ as does the $F_{X}(s)$ from the revPBE and it levels off at the same value as the $F_{X}(s)$ in the original PBE. The great similarity between $F_{X}(s)$ in the revPBE and the RPBE for $s$ values below 2.5 is resulting in very similar GGA contributions to the chemisorption energy, as can be judged from Figs. 2(b), 3(b), and 3(c) where the spatially and gradient-resolved contributions to $E_{\text {chem, } R P B E}-E_{\text {chem }, L D A}$ for $\mathrm{CO} / \mathrm{Pd}(100)$ have been plotted. From the RPBE based chemisorption energies given in Table III for the different adsorption systems it can be seen that the revPBE and the RPBE functionals generally give very similar results. When comparing the calculated chemisorption energies with the experimental ones, the rms deviations reported in Table III for the RPBE are thus as good as the ones for the revPBE. In other words, when focussing on the improved chemisorption energies, the RPBE functional represents an alternative to the revPBE. This also holds for the molecular bond energies [see the Appendix, issue (i)].

While improving the chemisorption energies, the revPBE and RPBE functionals may worsen other physical properties. This is apparent from Table I, showing revPBE and RPBE based Pd lattice constants that are $0.5-0.75 \%$ larger than with the PW91 and PBE functionals. These functionals already give lattice constants that are too large by about $2.5 \%$ compared to an experimental value of $2.89 \AA$. Another example is the worsening of LDA surface exchange energies ${ }^{45}$ by the PBE and presumably more so with the revPBE or RPBE.

We would like to stress that while the revPBE functional deviates from the PBE functional in the value of one parameter $\kappa$ in the exchange enhancement factor $F_{X}(s)$, the RPBE functional deviates from the PBE functional in the functional form of $F_{X}(s)$ itself. The selection in the revPBE of a larger $\kappa$ to some extent challenges the principles used in the derivation of the PBE- the Lieb-Oxford bound is no longer fulfilled by construction. The use of a different functional form for $F_{X}(s)$ in the RPBE, on the other hand, is as good a choice as the one made in the original PBE derivation. The differences in the chemisorption energies resulting from the PBE and RPBE functionals are therefore indicative of the uncertainty in the physical properties of a GGA functional resulting from the arbitrariness in the choice of functional form alone.

\section{SUMMARY}

In summary we have studied the chemisorption energies of atoms and molecules on late transition-metal surfaces. We have found that using the LDA functional for the exchangecorrelation description results in chemisorption energies that are numerically too large by about $1.5 \mathrm{eV}$ per adsorbate. Including nonlocal exchange-correlation effects with the PW91 or PBE functionals, this overbinding is reduced to approximately half an $\mathrm{eV}$ per adsorbate. The overbinding of the chemisorbed atoms and molecules is found to be further reduced by about a factor of two when using the revPBE functional suggested by Zhang and Yang or the RPBE functional suggested in the present work. The RPBE functional only differs from the PBE functional in the choice of the mathematical form for the exchange energy enhancement factor. The two functionals, PBE and RPBE, follow the same construction logic and therefore contain the same physics and fulfill the same physical criteria. 
TABLE IV. Molecular bond energies using oxygen pseudopotentials with $4.5 \%$ (PP-4.5\%-O core) and $42 \%$ (PP-42\%-O core) of the two $1 s$ core electrons. Also shown are all-electron (AE) results where available. The corresponding experimental values (with zero point energy removed) are 5.23, 11.24, and $6.63 \mathrm{eV}$ (Ref. 14).

\begin{tabular}{lcccccc}
\hline \hline & & LDA & PW91 & PBE & revPBE & RPBE \\
\hline & & & & & & \\
$\mathrm{O}_{2}$ & PP-4.5\%-O core & 7.10 & 5.84 & 5.81 & 5.53 & 5.51 \\
$\mathrm{O}_{2}$ & PP-42\%-O core & 7.30 & 6.06 & 5.99 & 5.63 & 5.59 \\
$\mathrm{O}_{2}$ & AE, Ref. 13 & 7.59 & 6.20 & 6.24 & & \\
$\mathrm{O}_{2}$ & AE, Ref. 14 & & & 6.14 & 5.75 & \\
& & & & & & \\
$\mathrm{CO}$ & PP-4.5\%-O core & 12.38 & 11.26 & 11.17 & 10.87 & 10.86 \\
$\mathrm{CO}$ & PP-42\%-O core & 12.54 & 11.40 & 11.28 & 10.93 & 10.90 \\
$\mathrm{CO}$ & AE, Ref. 13 & 12.96 & 11.66 & 11.66 & & \\
$\mathrm{CO}$ & AE, Ref. 14 & & & 11.60 & 11.19 & \\
& & & & & & \\
$\mathrm{NO}$ & PP-4.5\%-O core & 8.08 & 7.00 & 7.01 & 6.77 & 6.74 \\
$\mathrm{NO}$ & PP-42\%-O core & 8.23 & 7.13 & 7.11 & 6.82 & 6.78 \\
$\mathrm{NO}$ & AE, Ref. 13 & 8.63 & 7.41 & 7.46 & & \\
$\mathrm{NO}$ & AE, Ref. 14 & & & 7.36 & 6.96 & \\
\hline \hline
\end{tabular}

\section{ACKNOWLEDGMENTS}

This work was financed in part by The Danish Research Councils through The Center for Surface Reactivity and Grants Nos. 9501775 and 9800425 . The center for Atomicscale Materials Physics is sponsored by the Danish National Research Foundation.

\section{APPENDIX}

The calculational setup involves a number of approximations, some of which we have tested separately. These include (i) the use of pseudopotentials, (ii) the use of PW91 based pseudopotentials irrespective of the exchangecorrelation energy functional used in the calculations, (iii) the slab approximation, (iv) the rigid surface approximation, (v) the use of a finite coverage (1/4 ML), and (vi) the neglect of zero-point energy effects. Each of these issues are addressed below and we find that none of them affect the conclusions made in this work.

(i) The use of pseudopotentials. In Table IV we report our calculated molecular bond energies for $\mathrm{O}_{2}, \mathrm{CO}$, and $\mathrm{NO}$ with two different oxygen pseudopotentials (PP's) and the corresponding all-electron (AE) results from the literature. ${ }^{13,14}$ The molecular bond energies are seen to be somewhat different for the two oxygen pseudopotentials used and a general shift towards weaker molecular bond energies when using the pseudopotentials can be seen comparing to the AE results. One should, however, note that the $\mathrm{AE}$ results also disagree somewhat. We attribute the differences between the PP and AE results of the Table to the physical approximations made when using the pseudopotentials. This is supported by the finding that the molecular bond energies are sensitive to the details of the pseudopotential. The PP$4.5 \%$-O-core pseudopotential is an oxygen pseudopotential with a polynomial partial core inside $r_{c}^{O}=0.7$ bohr. It describes only $4.5 \%$ of the total core charge with the core den-

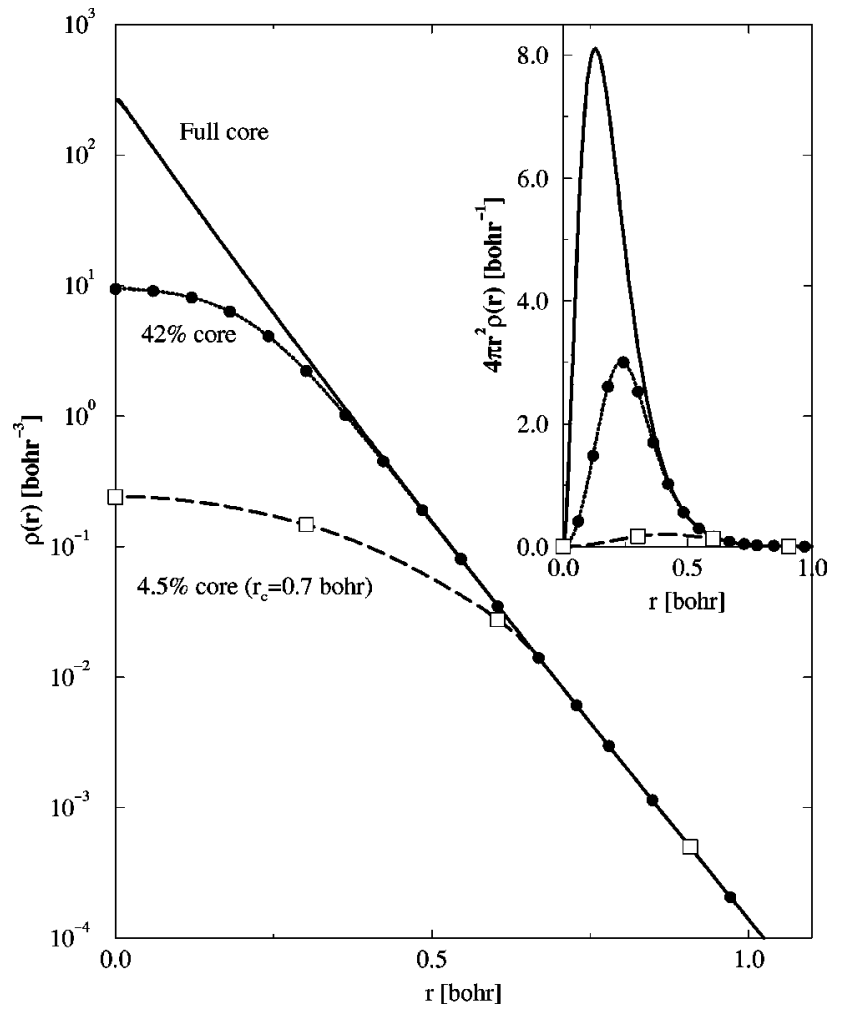

FIG. 4. The full all-electron core density and the partial core densities used in the PP-4.5\%-O-core and PP-42\%-O-core oxygen pseudopotentials. In the inset the spherical Jacobian factor $4 \pi r^{2}$ has been included. A denser sampling (black circles) is required for $\mathrm{PP}-42 \%-\mathrm{O}$ core compared to PP-4.5\%-O core (open squares).

sity being rather small at the oxygen core position. It may therefore cause unphysically large reduced density gradients, $\propto\left|\nabla \rho_{\sigma}\right| / \rho_{\sigma}^{4 / 3}$ for the minority spin, $\sigma$ in this region of space. To circumvent this we have constructed an improved oxygen pseudopotential, $\mathrm{PP}-42 \%-\mathrm{O}$ core, which retains $42 \%$ of the two oxygen core electrons. This is done by constructing the partial core density, ${ }^{20} \tilde{\rho}$, as $\tilde{\rho}(\mathrm{r})^{-1}=\rho_{0}^{-1}+\rho(\mathrm{r})^{-1}$, where $\rho$ is the all-electron core density and $\rho_{0}=125 /(4 \pi)$ bohr $^{-3}$ is a constant. The partial cores of the two pseudopotentials are shown in Fig. 4. As shown by Table IV the use of the PP$42 \%-\mathrm{O}$ core improves the molecular bond energies (using the AE results - not the experimental results - as the target values). The prize of using the more accurate pseudopotential is that the exchange-correlation energy integrals must be evaluated at more dense grids (as indicated in Fig. 4). In calculating the values in Tables I, II, and III we have therefore chosen during the force calculations and geometry optimization to use the PP-4.5\%-O-core pseudopotential, while only using the PP-42\%-O-core pseudopotential whenever evaluating the chemisorption energetics. While no direct comparison to AE chemisorption energies is possible, we can estimate the quality of the pseudopotential approximation by the sensitivity of the chemisorption energies to the oxygen pseudopotential used. If we use the PP-4.5\%-O-core pseudopotential the oxygen, $\mathrm{CO}$ and $\mathrm{NO}$ on $\mathrm{Pd}(111)$ chemisorption bond energies are reduced by $0.12(0.05), 0.01(<0.01)$ and $0.01(0.01) \mathrm{eV}$ in the PW91 (revPBE) compared to the values reported in Table III where the PP-42\%-O-core pseudopotential has been used. The chemisorption energies thus ap- 
pear to be much less sensitive to the pseudopotential approximation than do the molecular bond energies.

(ii) The pseudopotentials we use are constructed using the PW91 functional irrespective of the exchange-correlation functional used in the chemisorption energy evaluation. We have therefore conducted a set of self-consistent PW91 chemisorption energy calculations for $\mathrm{O} / \mathrm{Pd}(111)$ and $\mathrm{CO} /$ $\operatorname{Pd}(111)$ with $\mathrm{C}, \mathrm{O}$, and Pd pseudopotentials developed in the LDA approximation. We find that changing the pseudopotentials only causes the chemisorption energies to be reduced by $0.03 \mathrm{eV}$ and less than $0.01 \mathrm{eV}$ for the two chemisorption systems, respectively.

(iii) We approximate the surfaces by slabs of finite thickness. Extending the Pd(111) slab thickness from three to five close packed layers weakens the chemisorption bond of $\mathrm{O}$ and $\mathrm{CO}$ by $0.04 \mathrm{eV}$ and $0.02 \mathrm{eV}$, respectively.

(iv) We neglect the surface relaxation before and after the adsorption throughout the present work. Inclusion of such relaxations for the $\mathrm{O} / \mathrm{Ni}(100)$ system results in a weakening of the PW91 based oxygen chemisorption bond strength by $0.02 \mathrm{eV}$ (the clean surface gains more energy by the surface relaxation than does the oxygen covered surface, hence the bond is weakened).

(v) The present study only considers chemisorption at $1 / 4$ ML coverage, while we make a comparison to the initial, i.e., low coverage, experimental chemisorption energies. The use of the initial chemisorption energies is made in order to avoid the discussion of the most stable ordered adsorbate structures at finite coverage. Studies by Jennison, Schultz, and Sears ${ }^{46}$ suggest that for, e.g., the $\mathrm{CO}$ molecules at the separations in the 1/4 ML structure, the residual CO-CO interactions are at the $0.01 \mathrm{eV}$ level.

(vi) We omit the zero-point motion of the adsorbates. For the adsorption of atomic oxygen, we expect the perpendicular vibrational mode to be of the order $\hbar \omega=40-70 \mathrm{meV}$ (Ref. 47) and the parallel modes presumably smaller. This means that the theoretical chemisorption energies at most are affected by the order $0.05-0.1 \mathrm{eV}$ by zero-point motion. For the molecular $\mathrm{CO}$ and $\mathrm{NO}$ adsorbates, the intramolecular vibrational mode is softened somewhat upon adsorption, the mode of the entire molecule vibrating perpendicular to the surface is build up to about $\hbar \omega=60 \mathrm{meV}$ and the four frustrated translational and rotational modes to a few $\mathrm{meV} .{ }^{48}$ The changes are thus small and they even cancel to some degree and we therefore expect the zero point energy contribution to the $\mathrm{CO}$ and $\mathrm{NO}$ chemisorption energies to be very small.

While most of the above effects are found to be nonnegligible, they nonetheless appear small on the energy scale $0.25 \mathrm{eV}-1.0 \mathrm{eV}$, which, according to the present work, is relevant when comparing the density-functional theory derived chemisorption energies with experimental values.
${ }^{1}$ R. O. Jones and O. Gunnarsson, Rev. Mod. Phys. 61, 689 (1989).

${ }^{2}$ J. P. Perdew, J. A. Chevary, S. H. Vosko, K. A. Jackson, M. R. Pederson, D. J. Singh, and C. Fiolhais, Phys. Rev. B 46, 6671 (1992).

${ }^{3}$ X. J. Kong, C. T. Chan, K. M. Ho, and Y. Y. Ye, Phys. Rev. B 42, 9357 (1990).

${ }^{4}$ G. Ortiz, Phys. Rev. B 45, 11328 (1992).

${ }^{5}$ M. Körling and J. Häglund, Phys. Rev. B 45, 13293 (1992).

${ }^{6}$ A. Garcia, C. Elsässer, J. Zhu, S. G. Louie, and M. L. Cohen, Phys. Rev. B 46, 9829 (1992).

${ }^{7}$ D. L. Novikov, A. J. Freeman, N. E. Christensen, A. Svane, and C. O. Rodriquez, Phys. Rev. B 56, 7206 (1997).

${ }^{8}$ B. Hammer, K. W. Jacobsen, and J. K. Nørskov, Phys. Rev. Lett. 70, 3971 (1993).

${ }^{9}$ P. H. T. Philipsen, G. te Velde, and E. J. Baerends, Chem. Phys. Lett. 226, 583 (1994).

${ }^{10}$ P. Hu, D. A. King, S. Crampin, M.-H. Lee, and M. C. Payne, Chem. Phys. Lett. 230, 501 (1994).

${ }^{11}$ B. Hammer, M. Scheffler, K. W. Jacobsen, and J. K. Nørskov, Phys. Rev. Lett. 73, 1400 (1994).

${ }^{12}$ J. A. White, D. M. Bird, M. C. Payne, and I. Stich, Phys. Rev. Lett. 73, 1404 (1994).

${ }^{13}$ J. P. Perdew, K. Burke, and M. Ernzerhof, Phys. Rev. Lett. 77, 3865 (1996).

${ }^{14}$ Y. Zhang and W. Yang, Phys. Rev. Lett. 80, 890 (1998).

${ }^{15}$ E. H. Lieb and S. Oxford, Int. J. Quantum Chem. 19, 427 (1981).

${ }^{16} \mathrm{~J}$. P. Perdew, in Unified Theory of Exchange and Correlation Beyond the Local Density Approximation, Electronic Structure of Solids '91, edited by P. Ziesche and H. Eschrig (Akademie Verlag, Berlin, 1991), p. 11.

${ }^{17}$ J. P. Perdew, K. Burke, and M. Ernzerhof, Phys. Rev. Lett. 80, 891 (1998).
${ }^{18}$ M. C. Payne, M. P. Teter, D. C. Allan, T. A. Arias, and J. D. Joannopoulos, Rev. Mod. Phys. 64, 1045 (1992).

${ }^{19}$ D. H. Vanderbilt, Phys. Rev. B 41, 7892 (1990).

${ }^{20}$ S. G. Louie, S. Froyen, and M. L. Cohen, Phys. Rev. B 26, 1738 (1982).

${ }^{21}$ J. Neugebauer and M. Scheffler, Phys. Rev. B 46, 16067 (1992).

${ }^{22}$ G. Kresse and J. Forthmüller, Comput. Mater. Sci. 6, 15 (1996).

${ }^{23}$ F. W. Kutzler and G. S. Painter, Phys. Rev. Lett. 59, 1285 (1987).

${ }^{24}$ J. A. White and D. M. Bird, Phys. Rev. B 50, 4954 (1994).

${ }^{25}$ D. M. Ceperley and B. J. Alder, Phys. Rev. Lett. 45, 566 (1980); S. H. Vosko, L. Wilk, and M. Nusair, Can. J. Phys. 58, 1200 (1980).

${ }^{26}$ W. A. Brown, R. Kose, and D. A. King, Chem. Rev. 98, 797 (1998).

${ }^{27}$ Y. Y. Yeo, L. Vattuone, and D. A. King, J. Chem. Phys. 106, 1990 (1997).

${ }^{28}$ D. H. Wei, D. C. Skelton, and S. D. Kevan, Surf. Sci. 381, 49 (1997).

${ }^{29}$ R. A. Van Santen and M. Neurock, Catal. Rev. Sci. Eng. 37, 557 (1995).

${ }^{30}$ B. Hammer, Y. Morikawa, and J. K. Nørskov, Phys. Rev. Lett. 76, 2141 (1996).

${ }^{31}$ P. A. Gravil, D. M. Bird, and J. A. White, Phys. Rev. Lett. 77, 3933 (1996).

${ }^{32}$ S. Wilke and M. Scheffler, Phys. Rev. B 53, 4926 (1996).

${ }^{33}$ B. Hammer, O. H. Nielsen, and J. K. Nørskov, Catal. Lett. 43, 31 (1997).

${ }^{34}$ C. Stempfl and M. Scheffler, Phys. Rev. Lett. 78, 1500 (1997).

${ }^{35}$ B. Hammer and J. K. Nørskov, Phys. Rev. Lett. 79, 4441 (1997).

${ }^{36}$ A. Eichler and J. Hafner, Phys. Rev. Lett. 79, 4481 (1997).

${ }^{37}$ J. J. Mortensen, B. Hammer, and J. K. Nørskov, Phys. Rev. Lett. 80, 4333 (1998). 
${ }^{38}$ A. Bogicevic, J. Strömquist, and B. Lundqvist, Phys. Rev. B 57, 4289 (1998).

${ }^{39}$ M. Mavrikakis, B. Hammer, and J. K. Nørskov, Phys. Rev. Lett. 81, 2819 (1998).

${ }^{40}$ B. Hammer, Faraday Discuss. Chem. Soc. 110, 323 (1998).

${ }^{41}$ J. J. Mortensen, L. B. Hansen, B. Hammer, and J. K. Nørskov, J. Catal. (to be published).

${ }^{42}$ A. Zupan, K. Burke, M. Ernzerhof, and J. P. Perdew, J. Chem. Phys. 106, 10184 (1997).

${ }^{43}$ F. G. Cruz, K.-C. Lam, and K. Burke, J. Phys. Chem. A 102, 4911 (1998).

${ }^{44}$ http://www.fysik.dtu.dk/rpbe

${ }^{45}$ J. M. Pitarke and A. G. Equiluz, Phys. Rev. B 57, 6329 (1998).
${ }^{46}$ D. R. Jennison, P. A. Schultz, and M. P. Sears, Phys. Rev. Lett. 77, 4828 (1996).

${ }^{47}$ R. I. Masel, Principles of Adsorption and Reaction on Solid Surfaces (Wiley-Interscience, New York, 1996).

${ }^{48}$ J. Ellis, J. P. Toennies, and G. Witte, J. Chem. Phys. 102, 5059 (1995).

${ }^{49}$ H. Conrad, G. Ertl, J. Koch, and E. E. Latta, Surf. Sci. 43, 462 (1974).

${ }^{50}$ R. D. Ramsier, Q. Gao, H. Neergaard Waltenburg,k K.-W. Lee, O. W. Nooij, L. Lefferts, and J. T. Yates, Jr., Surf. Sci. 320, 209 (1994).

${ }^{51}$ H. B. Gray, Chemical Bonds: An Introduction to Atomic and Molecular Structure (University Science Books, Mill Valley, California, 1994). 